\title{
Stabilizing the thermal lattice Boltzmann method by spatial filtering
}

\author{
J. J. J. Gillissen* \\ Center for Environmental Sensing and Modeling (CENSAM) IRG Singapore-MIT Alliance for Research and Technology (SMART) Centre, \\ 3 Science Drive 2, Singapore 117543
}

(Received 13 July 2016; published 4 October 2016)

\begin{abstract}
We propose to stabilize the thermal lattice Boltzmann method by filtering the second- and third-order moments of the collision operator. By means of the Chapman-Enskog expansion, we show that the additional numerical diffusivity diminishes in the low-wavnumber limit. To demonstrate the enhanced stability, we consider a threedimensional thermal lattice Boltzmann system involving 33 discrete velocities. Filtering extends the linear stability of this thermal lattice Boltzmann method to 10-fold smaller transport coefficients. We further demonstrate that the filtering does not compromise the accuracy of the hydrodynamics by comparing simulation results to reference solutions for a number of standardized test cases, including natural convection in two dimensions.
\end{abstract}

DOI: 10.1103/PhysRevE.94.043302

\section{INTRODUCTION}

Lattice Boltzmann (LB) is a computational fluid dynamics (CFD) approach that discretizes the distribution function $f$ of the single-particle position and velocity $c$. This distribution function evolves according to the continuous Boltzmann equation [1-5],

$$
\frac{\partial f}{\partial t}+\nabla \cdot(c f)=\Omega
$$

In Eq. (1) the left-hand side represents the molecular streaming, i.e., molecules moving in straight lines, and the right-hand side accounts for intermolecular collisions. Conventional CFD methods solve moments of $f$, whose evolution equations are the corresponding moments of Eq. (1).

LB numerically approximates Eq. (1) by discretizing the time, the $D$-dimensional position space, and the $D$-dimensional velocity space. In standard LB, time and position space are discretized uniformly, with a time step $\Delta t$ and a mesh size $\Delta x$. The velocity space is discretized into $Q$ velocities, which are denoted $c_{\alpha}$, where the index $\alpha$ runs between 1 and $Q$. In the remainder of this paper all variables are scaled with the mesh size $\Delta x$, the time step $\Delta t$, and the gas constant $R=k_{B} / m$, where $k_{B}$ is the Boltzmann constant and $m$ is the molecular mass. The distribution function at time $t$, position $\boldsymbol{x}$, and velocity $\boldsymbol{c}_{\alpha}$ is denoted $f_{\alpha}(\boldsymbol{x}, t)$. In order for LB to provide a correct description of the hydrodynamics, the velocity set $\boldsymbol{c}_{\alpha}$ must be sufficiently extensive, such that $f_{\alpha}$ can correctly represent the moments of $f$ up to third order for isothermal flow and up to fourth order for thermal flow. The LB equation is the discretized version of Eq. (1) and reads:

$$
f_{\alpha}\left(\boldsymbol{x}+\boldsymbol{c}_{\alpha}, t+1\right)-f_{\alpha}(\boldsymbol{x}, t)=\Omega_{\alpha}(\boldsymbol{x}, t) .
$$

The left-hand side of Eq. (2) is the explicit Euler forward discretization of the molecular streaming. Since in the previously mentioned LB unit system the $\boldsymbol{c}_{\alpha}$ are integer vectors, the particles move exactly between two neighboring grid nodes during one time step. This property of "exact advection" in combination with the local nature of the collision operator $\Omega_{\alpha}$ makes LB accurate and computationally efficient. Apart from these advantages, the method is also facing some problems.

\footnotetext{
*jurriaangillissen@gmail.com
}

In this paper we address the stability problem that arises when the temperature $T$ is allowed to dynamically vary in LB [6,7]. This so-called thermal LB method is stable only for relatively large transport coefficients; i.e., viscosity and thermal conductivity. From a practical perspective, this problem can be circumvented by computing the temperature field using an alternative method, while fixing the temperature in LB, which is used to compute the mass and momentum fields only [8-13]. In this work we will not consider this "two population" method. Instead we treat the temperature as an intrinsic and dynamical part of the distribution function. One way to stabilize thermal LB is by diminishing the time step [14-18]. This so-called fractional step method requires interpolations to advance the streaming operator in time and compromises the exact advection property of the LB method. On the other hand, methods that stabilize thermal LB by modifying the collision operator are referred to as modified equation methods. An example of a modified equation method is increasing the number of discrete velocities beyond the requirement for correct hydrodynamics, which increases the computational expenses [6,19-24]. Thermal LB has also been stabilized by reducing the velocity set below this requirement $[14,25]$. The resulting inconsistencies in the hydrodynamics can be remedied by subtracting the finite difference discretizations of the corresponding error terms [25]. Some authors attribute the source for the instability in thermal LB to the lack of a global H-theorem; see, e.g., Ref. [26]. Accordingly, progress has been made to construct an H-theorem satisfying collision operators, referred to as "entropic LB" [27-29]. It is further noted that isothermal LB has also been stabilized through a reformulation of the collision operator based on velocity centered moments [30].

In this paper we follow the modified equation method and stabilize thermal LB by spatial filtering. It is obvious, however, that filtering $f_{\alpha}$ directly would introduce numerical diffusion, effectively enhancing the transport coefficients beyond the desired values. To remedy this situation we propose to only filter the second- and third-order moments of the collision operator. This means that only fluxes of momentum and stresses are filtered while the conserved moments are not directly affected by the procedure. Indirectly, however, the conserved moments are affected by filtering momentum and stress fluxes. By means of the Chapman-Enskog expansion, 
we show that this indirect affect is equivalent of adding a higher-order Laplacian to the governing equations of the macroscopic variables, which demonstrates the recovery of correct hydrodynamics in the low-wave-number limit. It is noted that the concept of adding a higher-order Laplacian, also known as hyperviscosity, has previously been used to stabilize simulations of turbulent flow; see, e.g., Ref. [31]. However, to the best of our knowledge, it has not yet been applied to LB simulations. One advancement of our approach is the implementation of this hyperviscosity. We apply a second-order filter to a fraction of the components (secondand third-order moments), which is computationally more efficient than applying a fourth-order filter to all components of the distribution function. It is further noted that the errors introduced by the interpolations in the fractional step method, described above, could in principle be analyzed and remedied in similar fashions, i.e., by the Chapman-Enskog expansion and by a fine-tuning of the interpolation operator, respectively. The present filtering method, however, offers two distinct advantages over the fractional step method. First, it uses a unit time step, which ensures the "exact advection" property of LB, which is the fundamental advantage of LB over other explicit advection schemes. Second, while the fractional step method involves spatial mixing of all LB components through interpolations, the present method only mixes the momentum flux and the stress flux moments though the filter operator, which is computationally less expensive.

The remainder of the paper is organized as follows. Section II presents the details of a specific thermal LB scheme that we use to demonstrate the effectiveness of the proposed filtering method. Section III studies the linear stability of this method, using the stability theory of Lallemand and Luo [32]. Section IV details the filter operator and shows that this operator enhances the linear stability of the thermal LB method. It also proves that the filtering does not compromise the macroscopic equations in the long-wave-number limit. Section V demonstrates correct hydrodynamics of the filtered thermal LB method for a number of test cases. Appendix A presents details of the specific thermal LB scheme that we use to demonstrate the effectiveness of the proposed filtering method. Appendix B reproduces the linear stability theory of Lallemand and Luo [32]. Appendix C outlines the Chapman-Enskog analysis on the effect of the filter on the macroscopic equations. Appendices D and E outline reference solutions to test cases.

\section{THE THERMAL LATTICE BOLTZMANN METHOD}

The purpose of this work is to present a concept for stabilizing thermal LB methods, which is spatial filtering the second- and third-order collision moments. The main result is a theoretical proof that this filtering procedure does not compromise the hydrodynamic equations in the long-wavenumber limit. To demonstrate the concept we apply it to a specific three-dimensional (3D) thermal lattice Boltzmann scheme, with $Q=33$ discrete velocities, referred to as D3Q33. The D3Q33 model incorporates discrete velocities with speeds $c_{\alpha}=\left|\boldsymbol{c}_{\alpha}\right|=0,1, \sqrt{2}, \sqrt{3}$, and 2 , corresponding to $1,6,12,8$, and 6 discrete velocities for each speed. It must be noted that the D3Q33 model lacks isotropy of lattice tensors of rank six and higher, resulting in anisotropic viscosity and heat conductivity [33-35]. However, these anisotropies diminish in the low-wave-number limit as $k^{2}$ (see, e.g., Ref. [36] and Fig. 2 below), which is similar as the effect of the filtering (see Fig. 4 below). Therefore, the use of D3Q33 does not compromise the accuracy of the method further than the filtering itself. It is further noted that the filtering operator can equally be applied to LB methods with larger velocity sets, without introducing any additional computational intensity or complexity. This is because the number (15) of filtered nonconserved second- and third-order collision moments does not change on increasing the velocity set.

To demonstrate the effectiveness of the proposed filtering method, we use the so-called multiple relaxation time (MRT) collision model, which replaces $\Omega_{\alpha}$ in Eq. (2) by a relaxation process that drives the moments of the distribution function to their equilibrium values using a set of separately tunable relaxation times $\tau_{\alpha}$ [37]. There are $Q$-independent moments $\tilde{f}_{\alpha}$. Five of these moments are conserved during collision, which are the mass density $\rho$, the three components of the momentum density $\rho \boldsymbol{u}$, and the energy density. All other moments are nonconserved. The moments are defined as weighted averaged polynomials $\phi_{\alpha \beta}$ of the velocity,

$$
\tilde{f}_{\alpha}=\phi_{\alpha \beta} f_{\beta} \text {. }
$$

The first index of $\phi_{\alpha \beta}$ indicates the polynomial and the second index indicates the velocity. Throughout this paper summation over repeated indices is assumed, unless stated otherwise. Furthermore, we use the tilde to indicate that a certain variable is represented in the moment space, as opposed to the velocity space. The polynomials for the current D3Q33 model are constructed using the standard Gram Schmidt procedure [38] and are reported in Appendix A. Since the polynomials form a complete orthogonal basis, the $\phi_{\alpha \beta}$ matrix is orthogonal with an inverse $\phi_{\alpha \beta}^{-1}$, which transforms the moment space into the velocity space,

$$
f_{\alpha}=\phi_{\alpha \beta}^{-1} \tilde{f}_{\beta}
$$

With the above-mentioned definitions the MRT collision operator is written as follows:

$$
\Omega_{\alpha}=\phi_{\alpha \beta}^{-1} \tilde{\Lambda}_{\beta \gamma}\left[\tilde{f}_{\gamma}^{(\mathrm{eq})}-\phi_{\gamma \delta} f_{\delta}\right] \text {. }
$$

Here $\tilde{f}_{\alpha}^{(\mathrm{eq})}$ are the equilibrium moments and $\tilde{\Lambda}_{\alpha \beta}$ is the collision matrix, evaluated in the moment space, which has the inverse relaxation times $\tau_{\alpha}^{-1}$ for the various moments on its diagonal. Perfect gas thermohydrodynamics requires that the equilibrium moments $\tilde{f}_{\alpha}^{(\mathrm{eq})}$ up to fourth order are identical to those of the Maxwell-Boltzmann distribution, which for the current D3Q33 model are listed in Appendix A. Since the higher-order moments have no effect on (long-wavelength) hydrodynamics, we relax these to zero $\left[\tilde{f}_{\alpha}^{(\text {eq })}=0\right]$ using $\tau_{\alpha}=1$. By applying the Chapman-Enskog expansion to the thermal LB model, the thermohydrodynamic equations for a perfect gas are recovered, with a dynamic viscosity of $\mu=$ $\rho T\left(\tau_{\mu}-\frac{1}{2}\right)$, a thermal conductivity of $\kappa=\rho T c_{p}\left(\tau_{\kappa}-\frac{1}{2}\right)$, a specific heat capacity at constant volume of $c_{v}=\frac{D}{2}$, a specific heat capacity at constant pressure of $c_{p}=c_{v}+1=\frac{D+2}{2}$, a specific heat capacity ratio of $\gamma=\frac{c_{p}}{c_{v}}=\frac{D+2}{D}$, a sound speed 
of $c_{s}=\sqrt{\gamma T}=\sqrt{\frac{D+2}{D} T}$, a kinematic viscosity of $\nu=\frac{\mu}{\rho}=$ $T\left(\tau_{\mu}-\frac{1}{2}\right)$, a thermal diffusivity of $\alpha=\frac{\kappa}{\rho c_{p}}=T\left(\tau_{\kappa}-\frac{1}{2}\right)$, and a Prandtl number of $\operatorname{Pr}=\frac{v}{\alpha}=\frac{\tau_{\mu}-\frac{1}{2}}{\tau_{\kappa}-\frac{1}{2}}$, where $\tau_{\mu}$ and $\tau_{\kappa}$ are the relaxation times for the second- and third-order moments, respectively; see, e.g., Ref. [6]. It is interesting that the $\frac{1}{2}$ in the expressions for the transport coefficients stems from second-order discretization errors of the LB scheme [Eq. (2)], which fortunately can be absorbed in the nonequilibrium (diffusive) momentum and heat fluxes; see, e.g., Ref. [6]. Chen et al. pointed out, that the energy equation derived from the thermal LB method with a diagonal collision matrix $\tilde{\Lambda}_{\alpha \beta}$ (in the moment space) contains a spurious, non-Galilean invariant term, when $\tau_{\mu} \neq \tau_{k}$, i.e., when the Prandtl number is other than one [39]. They cured this problem by designing a nondiagonal $\tilde{\Lambda}_{\alpha \beta}$ matrix. For simplicity, however, we do not adopt this approach, but we assume $\tau_{\mu}=\tau_{\kappa}=\tau$ instead, i.e., a Prandtl number of 1 . Finally, we note that the relaxation time for the fourth-order moment has no effect on hydrodynamics, and this value is set to 1 in the present study.

\section{INSTABILITY OF THE THERMAL LATTICE BOLTZMANN MODEL}

In this section we study the stability of the current thermal LB model using the linear stability theory that was introduced in Ref. [32]. The theory is also used to demonstrate the low-wave-number isotropy of the stress flux moments in the D3Q33 LB model, guaranteeing correct thermohydrodynamic behavior. For completeness, the derivation of this theory is reproduced in Appendix B. The theory provides the following evolution equation for the Fourier transformed distribution function $\hat{f}_{\alpha}(\boldsymbol{k}, t)$ :

$$
\hat{f}_{\alpha}(\boldsymbol{k}, t+1)=L_{\alpha \beta}(\boldsymbol{k}) \hat{f}_{\beta}(\boldsymbol{k}, t),
$$

where $\boldsymbol{k}$ is the wave vector and $L_{\alpha \beta}$ is the linearized evolution operator,

$$
L_{\alpha \beta}=F_{\alpha \gamma}\left(\delta_{\gamma \beta}+\Lambda_{\gamma \delta}\left[E_{\delta \beta}-\delta_{\delta \beta}\right]\right) .
$$

Here $\delta_{\alpha \beta}$ is the unit matrix, $F_{\alpha \beta}$ contains $\exp \left(-i \boldsymbol{k} \cdot \boldsymbol{c}_{\alpha}\right)$ on its diagonal, and $E_{\alpha \beta}=\left.\frac{\left.\delta f_{\delta}^{(e)}\right)}{\delta f_{\beta}}\right|_{f=f^{(0)}}$, where $f_{\alpha}^{(0)}$ is the uniform and steady background, which in the stability theory is perturbed by $\hat{f}_{\alpha}(\boldsymbol{k}, t) \exp \left(i \boldsymbol{k} \cdot \boldsymbol{c}_{\alpha}\right)$. The eigenvalues $z_{\alpha}$ of $L_{\alpha \beta}$ correspond to growth rates and oscillation frequencies of the various modes in the system. Stability requires $\left|z_{\alpha}\right| \leqslant 1$ for all modes, or, equivalently, $v_{\alpha}>0$, where the mode viscosity $v_{\alpha}$ is related to the real part Re of $z_{\alpha}$ by

$$
v_{\alpha}=-\frac{\operatorname{Re} \log z_{\alpha}}{k^{2}}
$$

In 3D there are only five modes prone to instability (at each $\boldsymbol{k}$ ), while the remaining modes always have positive viscosities. In the low-wave-number limit these five modes are linear combinations of the conserved moments. Therefore we refer to these modes as the "conserved modes".

To study the linear stability of the current D3Q33 thermal LB model we compute the eigenvalues of $L_{\alpha \beta}$ using MATHEMATICA software. In the stability analysis we restrict ourselves to a background temperature of $T_{0}=\frac{2}{5}$. We consider two cases,
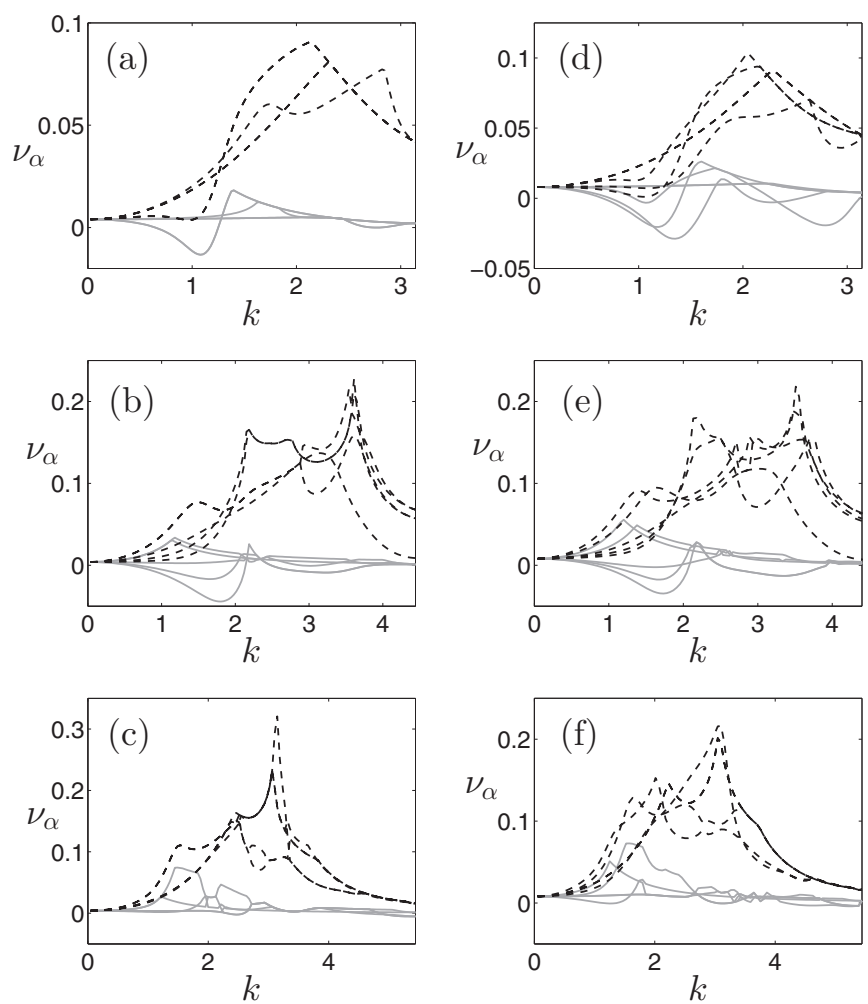

FIG. 1. Viscosity of conserved modes $v_{\alpha}$ as a function of absolute wave vector $k$ along $\boldsymbol{k}=k \boldsymbol{\delta}_{x}(\mathrm{a}, \mathrm{d}), \boldsymbol{k}=k \frac{1}{\sqrt{2}}\left(\boldsymbol{\delta}_{x}+\boldsymbol{\delta}_{y}\right)(\mathrm{b}, \mathrm{e})$, and $\boldsymbol{k}=k \frac{1}{\sqrt{3}}\left(\boldsymbol{\delta}_{x}+\boldsymbol{\delta}_{y}+\boldsymbol{\delta}_{z}\right)(\mathrm{c}, \mathrm{f})$. Data are shown for the unfiltered model (solid gray lines) and the filtered model (dashed black lines). In all cases the background temperature equals $T_{0}=\frac{2}{5}$. In (a)-(c) $\boldsymbol{u}_{0}=\mathbf{0}$ and $\tau-\frac{1}{2}=0.01$. In (d)-(f) $\boldsymbol{u}_{0}=\frac{c_{s} \boldsymbol{k}}{4 k}$ and $\tau-\frac{1}{2}=0.02$.

where the background velocity is either zero, $\boldsymbol{u}_{0}=0$, or a quarter sound speed $c_{s}=\sqrt{\frac{2}{3}} \approx 0.82$ along the direction of the wave vector $\boldsymbol{k}$, i.e., $\boldsymbol{u}_{0}=\frac{c_{s} \boldsymbol{k}}{4 k}$, where $k$ is the absolute value of $\boldsymbol{k}$. In the latter case we choose to align $\boldsymbol{u}_{0}$ with $\boldsymbol{k}$, since it is known that this renders the model most unstable, see, e.g., Ref. [32]. We find that without flow the model is unstable when $\tau-\frac{1}{2} \lesssim 0.05$ and a quarter sound speed extends the instability region to $\tau-\frac{1}{2} \lesssim 0.1$. The gray lines in Fig. 1 show the viscosity of the conserved modes of the current (unfiltered) model. The mode viscosities are displayed as a function of the absolute value $k$ of $\boldsymbol{k}$, which is varied along three directions. Figures 1 (a) -1 (c) correspond to $\boldsymbol{u}_{0}=0$ and $\tau-\frac{1}{2}=0.01$, while Figs. 1(d) -1 (f) correspond to $\boldsymbol{u}_{0}=\frac{c_{s} \boldsymbol{k}}{4 k}$ and $\tau-\frac{1}{2}=0.02$. Instability $\left(v_{\alpha}<0\right)$ is seen not only in the upper region of the wave-number space but also in regions of intermediate $k$.

Second, we use the stability theory to study the isotropy of the D3Q33 LB model. For this purpose we use a background temperature of $T_{0}=\frac{2}{5}$, a background velocity of $\boldsymbol{u}_{0}=0$, and a relaxation time of $\tau-\frac{1}{2}=0.02$. We start by fixing the absolute wave vector $k=0.04$ and vary the orientation of $\boldsymbol{k}$ between the $x$ and the $y$ directions as $\boldsymbol{k}=k\left[\cos (\theta) \boldsymbol{\delta}_{x}+\sin (\theta) \boldsymbol{\delta}_{y}\right]$, where the angle $\theta$ is varied between 0 and $\pi / 2$. Figure 2(a) shows the relative viscosity variation $\left(v_{\alpha}-\left\langle v_{\alpha}\right\rangle_{\theta}\right) /\left\langle v_{\alpha}\right\rangle_{\theta}$ of the third-order, stress flux 

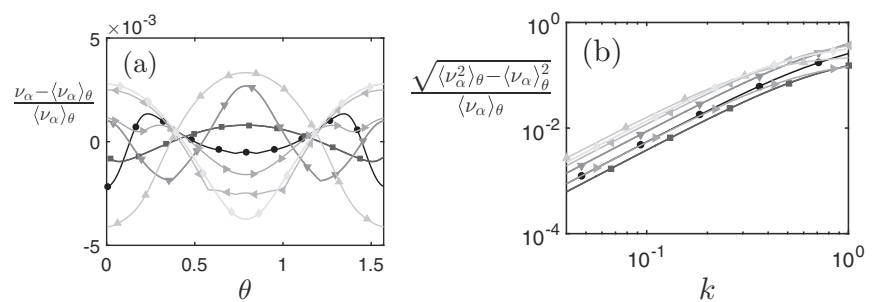

FIG. 2. (a) Relative viscosity variation $\left(v_{\alpha}-\left\langle v_{\alpha}\right\rangle_{\theta}\right) /\left\langle v_{\alpha}\right\rangle_{\theta}$ of the stress flux modes as a function of the orientation angle $\theta$ of the wave vector $\boldsymbol{k}=k\left[\cos (\theta) \boldsymbol{\delta}_{x}+\sin (\theta) \boldsymbol{\delta}_{y}\right]$ at a fixed absolute value $k=0.04$ of the wave vector. Here $\langle\cdot\rangle_{\theta}$ is the average of - over $\theta$. (b) Root-meansquare of the orientational viscosity variation $\sqrt{\left\langle v_{\alpha}^{2}\right\rangle_{\theta}-\left\langle v_{\alpha}\right\rangle_{\theta}^{2}} /\left\langle v_{\alpha}\right\rangle_{\theta}$ of the stress flux modes as a function of the absolute value $k$ of the wave vector $\boldsymbol{k}=k\left[\cos (\theta) \boldsymbol{\delta}_{x}+\sin (\theta) \boldsymbol{\delta}_{y}\right]$. The orientational variations diminish as $k^{2}$.

modes, where $\alpha=11 \cdots 20$. Here $\langle\cdot\rangle_{\theta}$ is the average of over $\theta$. The orientational variation is defined as the relative root-mean-square $\sqrt{\left\langle v_{\alpha}^{2}\right\rangle_{\theta}-\left\langle v_{\alpha}\right\rangle_{\theta}^{2}} /\left\langle v_{\alpha}\right\rangle_{\theta}$, which for the case in Fig. 2(a) is of the order of $10^{-3}$. Figure 2(b) shows that the orientational variations $\sqrt{\left\langle v_{\alpha}^{2}\right\rangle_{\theta}-\left\langle v_{\alpha}\right\rangle_{\theta}^{2}} /\left\langle v_{\alpha}\right\rangle_{\theta}$ diminish as $k^{2}$, demonstrating isotropy of the D3Q33 model in the lowwave-number limit, which confirms the theoretical prediction in Ref. [36].

\section{STABILIZING THE THERMAL LATTICE BOLTZMANN MODEL BY SPATIAL FILTERING}

We enhance the stability of the thermal lattice Boltzmann method by spatial filtering the second- and third-order moments of the collision operator. The filtered LB scheme is written as follows:

$$
\begin{aligned}
& f_{\alpha}\left(\boldsymbol{x}+\boldsymbol{c}_{\alpha}, t+1\right) \\
& =f_{\alpha}(\boldsymbol{x}, t)+\phi_{\alpha \beta}^{-1} \sum_{\boldsymbol{y}}\left[\delta_{\beta \gamma} \delta(\boldsymbol{y})+v^{F} \tilde{N}_{\beta \gamma} \psi(\boldsymbol{y})\right] \\
& \quad \times \tilde{\Lambda}_{\gamma \delta}\left[\tilde{f}_{\delta}^{(\mathrm{eq})}(\boldsymbol{x}+\boldsymbol{y}, t)-\phi_{\delta \epsilon} f_{\epsilon}(\boldsymbol{x}+\boldsymbol{y}, t)\right] .
\end{aligned}
$$

Here $\sum_{\boldsymbol{y}}\left(\delta_{\alpha \beta} \delta(\boldsymbol{y})+v^{F} \tilde{N}_{\alpha \beta} \psi(\boldsymbol{y})\right)$ is the filter operator, $v^{F}$ is the filter viscosity, and the function $\delta(\boldsymbol{y})$ is referred to as the "discrete delta function." It is one when $\boldsymbol{y}=\mathbf{0}$ and it is zero elsewhere. The matrix $\tilde{N}_{\alpha \beta}$ has unit diagonal elements for the nonconserved second- and third-order moments and is zero elsewhere. The nonconserved second- and third-order collision moments are convoluted with $\delta(\boldsymbol{y})+v^{F} \psi(\boldsymbol{y})$, while other collision moments are left alone by the filter operator, i.e., they are convoluted with $\delta(\boldsymbol{y})$.

We use a filter function $\psi(\boldsymbol{y})$ that involves a minimum number of neighbors, which is seven in $3 \mathrm{D}$; i.e., one at distance $y=0$ and six at distance $y=1$. This filter does not compromise the local nature of the LB method as these neighbors were already involved in the streaming operator. Furthermore, the filter contributes marginally to overall computational expenses, which are in fact mainly due to the transformations between the moment space and the velocity space. To substantiate this claim we note that the number of

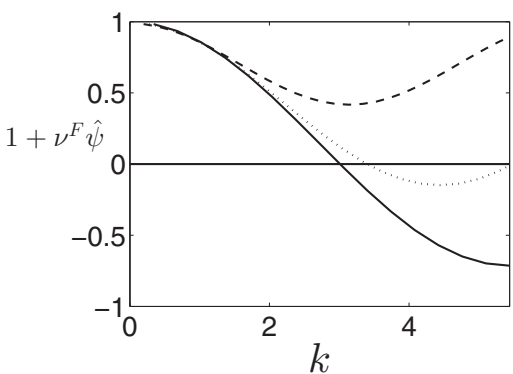

FIG. 3. Discrete Fourier transformed filter $1+v^{F} \hat{\psi}$ as a function of absolute wavenumber $k$ using $v^{F}=\frac{1}{15}$ for $\boldsymbol{k}=k \boldsymbol{\delta}_{x}$ (dashed line), $\boldsymbol{k}=k \frac{1}{\sqrt{2}}\left(\boldsymbol{\delta}_{x}+\boldsymbol{\delta}_{y}\right)$ (dotted line), and $\boldsymbol{k}=k \frac{1}{\sqrt{3}}\left(\boldsymbol{\delta}_{x}+\boldsymbol{\delta}_{y}+\boldsymbol{\delta}_{z}\right)$ (solid line).

multiplications that are required by the filtering routine per lattice node and per time step equals 7 (number of nodes in filter stencil) times 15 (number of nonconserved secondand third-order moments) equals 90, while for the the present D3Q33 the transformations between moment and velocity space requires 2 (transform back and forth) times $33^{2}$ (number of matrix elements) equals 2178 multiplications.

Symmetry and conservation require that the $3 \mathrm{D}$ filter function has the following form:

$$
\psi(y)= \begin{cases}-6 & \text { if } y=0 \\ 1 & \text { if } y=1\end{cases}
$$

In Appendix $\mathrm{C}$ we employ the Chapman-Enskog expansion to demonstrate that the filtering replaces $\tau$ by $\tau\left(1-v^{F} \nabla^{2}\right)$ in the overall equations of fluid motion. This means that in the filtered model the kinematic viscosity (and thermal diffusivity) becomes wavelength dependent,

$$
v=v_{0}\left(1+\frac{\tau \nu^{F} k^{2}}{\tau-\frac{1}{2}}\right),
$$

where $v_{0}=T\left(\tau-\frac{1}{2}\right)$ is the kinematic viscosity at $k=0$. This result indicates that the diffusion induced by the filter diminishes in the low-wave-number limit, ensuring correct hydrodynamics.

The Chapman-Enskog expansion is valid only for small wave numbers. To study the effect of filtering on the whole wave number range, we apply linear stability theory to Eq. (9), which results in the following linearized evolution operator:

$$
L_{\alpha \beta}=F_{\alpha \gamma}\left\{\delta_{\gamma \beta}+\left[\delta_{\gamma \delta}+v^{F} N_{\gamma \delta} \hat{\psi}(\boldsymbol{k})\right] \Lambda_{\delta \epsilon}\left[E_{\epsilon \beta}-\delta_{\epsilon \beta}\right]\right\},
$$

where the convolution theorem is used to replace the Fourier transformed convolution of $\psi(\boldsymbol{x})$ and $f(\boldsymbol{x})$ by the product of their Fourier transforms $\hat{\psi}(\boldsymbol{k})$ and $\hat{f}(\boldsymbol{k})$. The discrete Fourier transformed filter function $1+v^{F} \hat{\psi}(\boldsymbol{k})$ is displayed in Fig. 3 for $v^{F}=\frac{1}{15}$ along three directions in the $\boldsymbol{k}$ space.

As in Sec. II we now analyze the stability of the filtered model using a background temperature of $T_{0}=\frac{2}{5}$, and we consider two cases, where the background velocity is either zero, $\boldsymbol{u}_{0}=0$, or a quarter sound speed along the direction of the wave vector, i.e., $\boldsymbol{u}_{0}=\frac{c_{s} \boldsymbol{k}}{4 k}$. The filter viscosity is chosen as $v^{F}=\frac{1}{15}$. In both cases we find that the filtered model has a larger stability region than the unfiltered model. For $\boldsymbol{u}_{0}=0$, 

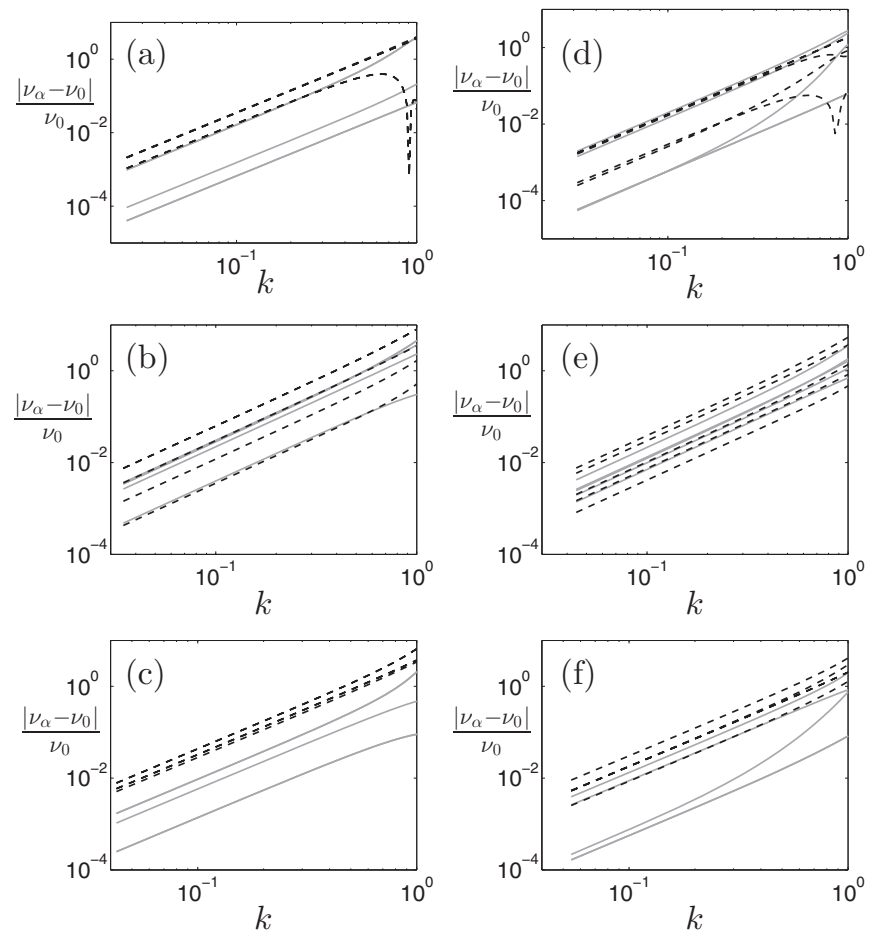

FIG. 4. The relative difference between the conserved mode viscosity $v_{\alpha}$ and the viscosity $v_{0}$ at zero wave number: $\left|v_{\alpha}-v_{0}\right| / v_{0}$ as a function of the absolute wave vector $k$ along $\boldsymbol{k}=k \boldsymbol{\delta}_{x}(\mathrm{a}, \mathrm{d})$, $\boldsymbol{k}=k \frac{1}{\sqrt{2}}\left(\boldsymbol{\delta}_{x}+\boldsymbol{\delta}_{y}\right)(\mathrm{b}, \mathrm{e})$, and $\boldsymbol{k}=k \frac{1}{\sqrt{3}}\left(\boldsymbol{\delta}_{x}+\boldsymbol{\delta}_{y}+\boldsymbol{\delta}_{z}\right)(\mathrm{c}, \mathrm{f})$. Data are shown for the unfiltered model (solid gray lines) and the filtered model (dashed black lines). In all cases the background temperature equals $T_{0}=\frac{2}{5}$. In (a)-(c) $\boldsymbol{u}_{0}=\mathbf{0}$ and $\tau-\frac{1}{2}=0.01$. In (d)-(f) $\boldsymbol{u}_{0}=\frac{c_{s} \boldsymbol{k}}{4 k}$ and $\tau-\frac{1}{2}=0.02$.

the stability region is extended from $\tau-\frac{1}{2} \gtrsim 0.05$ for the unfiltered model to $\tau-\frac{1}{2} \gtrsim 0.005$ for the filtered model. For $\boldsymbol{u}_{0}=\frac{c_{s} \boldsymbol{k}}{4 k}$ it is extended from $\tau-\frac{1}{2} \gtrsim 0.1$ to $\tau-\frac{1}{2} \gtrsim 0.015$. In both cases filtering results in a roughly 10 -fold decrease in permissible transport coefficients.

The dashed black lines in Fig. 1 show the viscosity of the conserved modes of the filtered, linearized evolution operator. Again, the data are presented along three directions in the $\boldsymbol{k}$ space. Figures 1(a)-1(c) correspond to $\boldsymbol{u}_{0}=0$ and $\tau-\frac{1}{2}=$ 0.01 , while Figs. 1(d) -1 (f) correspond to $\boldsymbol{u}_{0}=\frac{c_{s} \boldsymbol{k}}{4 k}$ and $\tau$ $\frac{1}{2}=0.02$. In contrast to the unfiltered model, all modes are stable $\left(v_{\alpha}>0\right)$ in the filtered model for the cases considered in Fig. 1.

In Fig. 4 we verify the predicted diminishing impact of the filtering on the low-wave-number behavior [Eq. (11)]. The figure shows the relative difference between the conserved mode viscosity $v_{\alpha}$ and the zero-wave-number viscosity: $v_{0}=$ $T\left(\tau-\frac{1}{2}\right)$. Data are displayed along different directions in $\boldsymbol{k}$ space for both the unfiltered and filtered model and for both $\boldsymbol{u}_{0}=0$ and $\boldsymbol{u}_{0}=\frac{c_{s} \boldsymbol{k}}{4 k}$. In agreement with Eq. (11), the $v_{\alpha}$ for both the unfiltered as well as the filtered model follow $k^{2}$. The prefactor, however, differs from Eq. (11). This is due to spurious higher-order terms in the Chapman-Enskog expansion, which also produce $k^{2}$-dependent mode viscosities; see, e.g., Ref. [36] and Fig. 2(b) above. It is seen from Figs. 2(b)
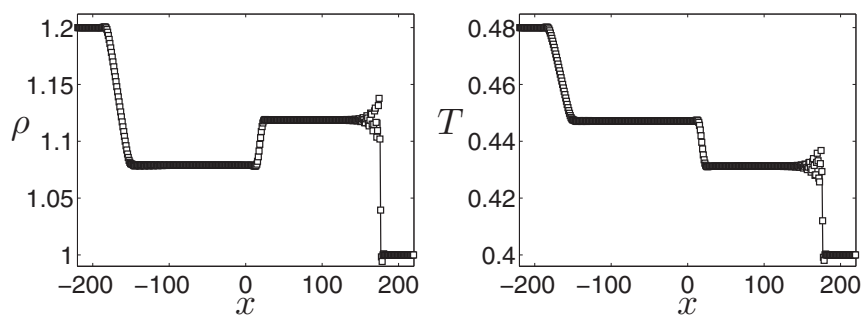

FIG. 5. Density $\rho$ and temperature $T$ as functions of the distance $x$ to the center of Sod's shock tube at time $t=200$ after removing the diaphragm, computed with the filtered thermal lattice Boltzmann model (markers) and reference solution (lines) presented in Ref. [40].

and Fig. 4 that in the current D3Q33 model the contribution from the spurious higher-order terms are of similar magnitude as that of the filtering.

\section{VALIDATING THE STABILIZED THERMAL LATTICE BOLTZMANN MODEL}

Finally, we validate that the proposed filtering does not compromise the long-wavelength hydrodynamics of the thermal lattice Boltzmann model. For this purpose we apply the filtered D3Q33 thermal LB model with $v_{F}=\frac{1}{15}$ to four test cases. The test cases are computed using a FORTRAN implementation of the LB method [Eq. (9)]. The first test case is Sod's one-dimensional shock tube [40]. In this case a gas at equilibrium with relaxation time 0.525 is contained within a closed tube between $x=-220$ and $x=220$. A diaphragm at $x=0$ separates the gas on the left which has a density of 1.2 and a temperature of 0.48 from the gas on the right which has a density of 1 and a temperature of 0.4 . At $t=0$, the diaphragm is removed and an intricate shock pattern develops, with a well-known analytical solution [40]. Figure 5 shows the density and the temperature distributions at $t=200$. Besides the usual wiggles, observed at $x=c_{s} t \approx 163$, the simulation agrees well with the corresponding analytical solution.

Second, we consider a thermal Poiseuille flow, where a gas with a relaxation time of 0.525 and an average mass density of 1 is driven in the $x$ direction between two walls that are located at $y=-8$ and $y=8$ and that are kept at a constant temperature of 0.4. The flow is driven by an external pressure gradient $-\nabla P=-\frac{d P}{d x} \delta_{x}$, where $-\frac{d P}{d x}=10^{-4}$. The pressure gradient is implemented by adding a source term: $-\phi_{\alpha \beta}^{-1} \frac{d P}{d x} \delta_{\beta 2}$ to Eq. (9). The no-slip boundary condition is enforced by the halfway bounce-back method. The constant temperature boundary condition is satisfied by adjusting the temperature $T_{z=\frac{1}{2}}$ at each node next to a wall, such that the temperature $T_{z=0}$, extrapolated from $T_{z=\frac{1}{2}}$ and $T_{z=\frac{3}{2}}$, equals the desired value at the wall,

$$
T_{z=\frac{1}{2}}=\frac{1}{3} T_{z=\frac{3}{2}}+\frac{2}{3} T_{z=0} .
$$

Due to viscous dissipation, a temperature distribution sets in between the walls. The simulated fully developed velocity and temperature distributions are presented in Fig. 6, which agree well with the reference solution, which is outlined in Appendix D. 

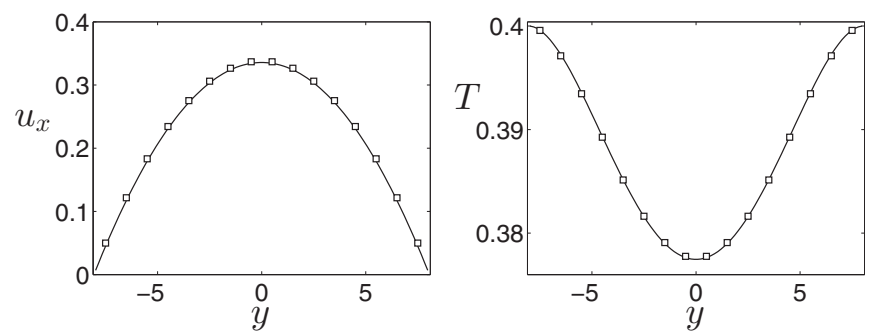

FIG. 6. Fully developed velocity $u_{x}$ and temperature $T$ as functions of the distance $y$ from the center line in thermal Poiseuille flow, computed with the filtered thermal lattice Boltzmann model (markers) and reference solution (lines) presented in Appendix D.

The third test case pertains to the one-dimensional Rayleigh Bénard setup, which does not involve convection. Here a gas with a relaxation time of 1 and an average density of 1 resides between a hot wall at $x=0$ with a temperature of 0.42 and a cold wall at $x=12$ with a temperature of 0.38 . The gravitational acceleration of $6.67 \times 10^{-3}$ points towards the hot wall in the negative $x$ direction. The simulated, fully developed density and temperature distributions in Fig. 7 agree well with the corresponding reference solution, which is outlined in Appendix E.

The fourth and final test case is that of two-dimensional Rayleigh Bénard convection [42]. The flow is driven by a hot bottom plate with temperature $T_{h}=T_{0}+\Delta T / 2$ and a cold top plate with temperature $T_{c}=T_{0}-\Delta T / 2$ and gravity $g$, which points in the negative $y$ direction. The heat flux $\Phi$ nondimensionalized by the conductive heat transfer $\frac{k \Delta T}{L}$ is referred to as the Nusselt number $\mathrm{Nu}=\frac{\Phi L}{\kappa \Delta T}$. The driving force in the system is measured by the nondimensional Rayleigh number $\operatorname{Ra}=\frac{\beta g \Delta T L^{3}}{\nu \alpha}$. Here $\beta$ is the thermal expansion coefficient, which for an ideal gas (with unit gas constant) equals $\beta=\frac{1}{T_{0}}$. Below a critical Rayleigh number of around 1708 the heat transfer is purely conductive, which corresponds to $\mathrm{Nu}=1$ [8]. Above the critical Rayleigh number, so-called Rayleigh-Bénard convection sets in, which enhances the heat transfer between the hot and cold walls, and $\mathrm{Nu}$ becomes an increasing function of Ra. We simulate the system above the critical Rayleigh number, again using an average background temperature of $T_{0}=0.4$. Periodic boundary conditions are applied in the horizontal $x$ direction. In order to facilitate a comparison to reference data $[8,41]$, we need to satisfy
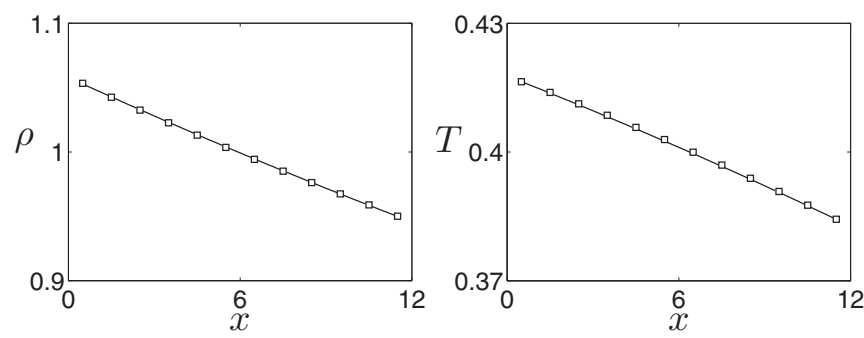

FIG. 7. Fully developed density $\rho$ and temperature $T$ as functions of the distance $x$ from the hot wall in the stationary Rayleigh Bénard setup, computed with the filtered thermal lattice Boltzmann model (markers) and reference solution (lines) presented in Appendix E.
TABLE I. Parameters for the 2D Rayleigh Bénard convection simulations.

\begin{tabular}{lcccc}
\hline \hline $\mathrm{Ra}$ & $L_{x}$ & $L_{y}$ & $g$ & $\tau$ \\
\hline 5000 & 256 & 128 & $3.125 \times 10^{-5}$ & 0.5286 \\
10000 & 512 & 256 & $1.563 \times 10^{-5}$ & 0.5405 \\
20000 & 768 & 384 & $1.042 \times 10^{-5}$ & 0.5429 \\
\hline \hline
\end{tabular}

(nearly) incompressible conditions, and therefore we use a small temperature difference $\Delta T / T_{0}=10^{-2}$ and a small gravitational acceleration $g=10^{-2} T_{0} / L$. Three cases are simulated using different values for the Rayleigh number, i.e., $\mathrm{Ra}=5000,10000$, and 20000 , respectively. In Table I the values for the numerical parameters are given. After starting from a linear temperature profile and a slightly perturbed velocity profile, the simulation undergoes a transient period. As the velocity magnitude is kept small, to satisfy above conditions, the simulation takes $\sim 10^{6}$ time steps in order to reach the steady state. Figure 8 shows contour plots of the steady-state temperature distribution, correctly showing that, with increasing $\mathrm{Ra}$, there is an increased convective mixing and an increased temperature gradient near the wall.

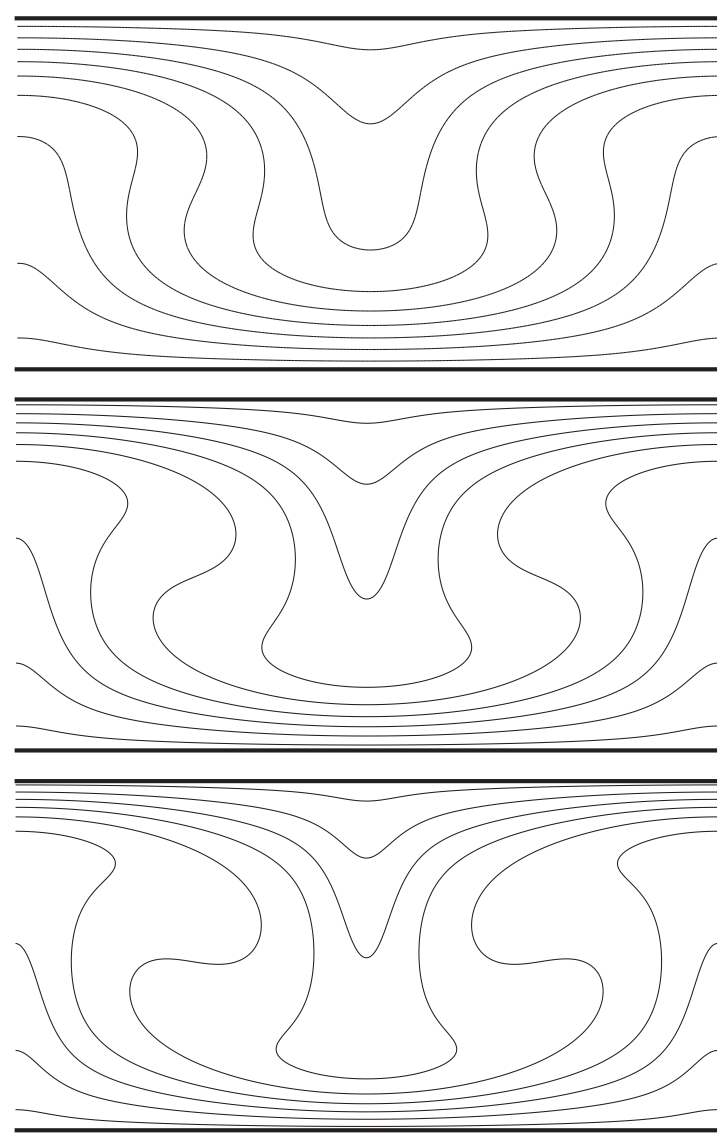

FIG. 8. Temperature distribution in Rayleigh Bénard convection for three Rayleigh numbers of $\mathrm{Ra}=5000$ (top), $\mathrm{Ra}=10000$ (middle), and $\mathrm{Ra}=20000$ (bottom), visualized using 10 temperature contours that are equally spaced between the bottom temperature of 0.402 and the top temperature of 0.398 . The domain dimensions and other parameters are reported in Table I. 


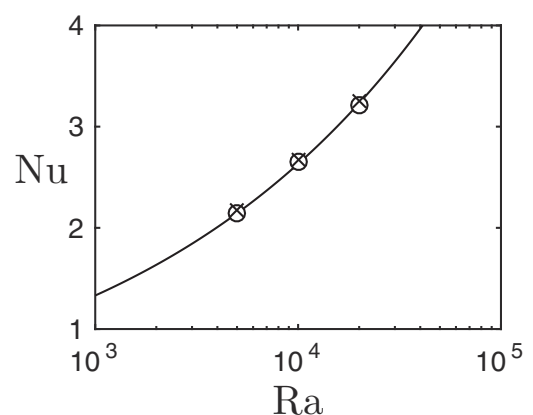

FIG. 9. The Nusselt number $\mathrm{Nu}$ as a function of the Rayleigh number Ra in Rayleigh Bénard convection, obtained from the present simulations (crosses), reference values [41] (circles), and an empirical formula $\mathrm{Nu}=1.56\left(\mathrm{Ra} / \mathrm{Ra}_{c}\right)^{0.296}$, where the critical Rayleigh number $\mathrm{Ra}_{c}=1708$ [8].

The heat transfer is numerically computed using:

$$
\Phi=\frac{\kappa}{2}\left(T_{h}-\langle T\rangle_{1}+\langle T\rangle_{L_{y}}-T_{c}\right),
$$

where $\langle T\rangle_{1}$ and $\langle T\rangle_{L_{y}}$ are the temperatures averaged over $x$ on the nearest grid line next to the lower and upper walls, respectively, i.e., $\langle T\rangle_{j}=L_{y}^{-1} \sum_{i=1}^{L_{x}} T_{i, j}$. The resulting, simulated Nusselt number is presented as a function of the Rayleigh number in Fig. 9. The results are in very good agreement with the reference solutions $[8,41]$.

\section{CONCLUSIONS}

We show that thermal LB can be stabilized by filtering the second- and third-order collision moments, effectively diffusing the nonequilibrium momentum and heat fluxes, and thereby introducing additional $k^{2}$-dependent diffusion terms to the hydrodynamic equations. The $k^{2}$-dependent diffusion terms are of the same order of magnitude as the spurious higher-order terms in the Chapman-Enskog expansion of the current D3Q33 LB method. Therefore the filtering does not compromise the accuracy of this method.

The limitation of the proposed filter [Eq. (10)] is related to its wave-number dependence $\hat{\psi}(\boldsymbol{k})$, displayed in Fig. 3. Below a critical $\tau-\frac{1}{2}$, the amount of $v^{F} \hat{\psi}(\boldsymbol{k})$ needed to stabilize intermediate $\boldsymbol{k}$ corresponds to destabilizing $v^{F} \hat{\psi}(\boldsymbol{k})$ values at large $\boldsymbol{k}$. Although, beyond the scope of the present work, the stabilization range of the method can be improved by extending the filter stencil, thereby tailoring the shape of $\hat{\psi}(\boldsymbol{k})$.

\section{APPENDIX A: BASIS FUNCTIONS AND EQUILIBRIUM MOMENTS FOR THE D3Q33 MODEL}

The basis functions of the D3Q33 model are as follows:

$$
\begin{aligned}
\phi_{1 \alpha} & =1, \\
\phi_{2 \alpha} & =c_{\alpha x}, \\
\phi_{3 \alpha} & =c_{\alpha y}, \\
\phi_{4 \alpha} & =c_{\alpha z}, \\
\phi_{5 \alpha} & =-26+11 c_{\alpha}{ }^{2}, \\
\phi_{6 \alpha} & =-c_{\alpha}{ }^{2}+3 c_{\alpha x}{ }^{2},
\end{aligned}
$$

$$
\begin{array}{rl}
\phi_{7 \alpha}= & c_{\alpha y}{ }^{2}-c_{\alpha z}{ }^{2}, \\
\phi_{8 \alpha}= & c_{\alpha x} c_{\alpha y}, \\
\phi_{9 \alpha}= & c_{\alpha x} c_{\alpha z}, \\
\phi_{10 \alpha}= & c_{\alpha y} c_{\alpha z}, \\
\phi_{11 \alpha}= & -37 c_{\alpha x}+13 c_{\alpha}{ }^{2} c_{\alpha x}, \\
\phi_{12 \alpha}= & -37 c_{\alpha y}+13 c_{\alpha}{ }^{2} c_{\alpha y}, \\
\phi_{13 \alpha}= & -37 c_{\alpha z}+13 c_{\alpha}{ }^{2} c_{\alpha z}, \\
\phi_{14 \alpha}= & c_{\alpha x} c_{\alpha y}{ }^{2}-c_{\alpha x} c_{\alpha z}{ }^{2}, \\
\phi_{15 \alpha}= & -c_{\alpha x}{ }^{2} c_{\alpha y}+c_{\alpha y} c_{\alpha z}{ }^{2}, \\
\phi_{16 \alpha}= & c_{\alpha x}{ }^{2} c_{\alpha z}-c_{\alpha y}{ }^{2} c_{\alpha z}, \\
\phi_{17 \alpha}= & 55 c_{\alpha x}-45 c_{\alpha}{ }^{2} c_{\alpha x}+38 c_{\alpha x}{ }^{3}, \\
\phi_{18 \alpha}= & 55 c_{\alpha y}-45 c_{\alpha}{ }^{2} c_{\alpha y}+38 c_{\alpha y}{ }^{3}, \\
\phi_{19 \alpha}= & 55 c_{\alpha z}-45 c_{\alpha}{ }^{2} c_{\alpha z}+38 c_{\alpha z}{ }^{3}, \\
\phi_{20 \alpha}= & c_{\alpha x} c_{\alpha y} c_{\alpha z}, \\
\phi_{21 \alpha}= & 304-325 c_{\alpha}{ }^{2}+69 c_{\alpha}{ }^{4}, \\
\phi_{22 \alpha}= & 69 c_{\alpha}{ }^{2}-19 c_{\alpha}{ }^{4}-207 c_{\alpha x}{ }^{2}+57 c_{\alpha}{ }^{2} c_{\alpha x}{ }^{2}, \\
\phi_{23 \alpha}= & -69 c_{\alpha y}{ }^{2}+19 c_{\alpha}{ }^{2} c_{\alpha y}{ }^{2}+69 c_{\alpha z}{ }^{2}-19 c_{\alpha}{ }^{2} c_{\alpha z}{ }^{2}, \\
\phi_{24 \alpha}= & -8 c_{\alpha x} c_{\alpha y}+3 c_{\alpha}{ }^{2} c_{\alpha x} c_{\alpha y}, \\
\phi_{25 \alpha}= & -8 c_{\alpha x} c_{\alpha z}+3 c_{\alpha}{ }^{2} c_{\alpha x} c_{\alpha z}, \\
\phi_{26 \alpha}= & -8 c_{\alpha y} c_{\alpha z}+3 c_{\alpha}{ }^{2} c_{\alpha y} c_{\alpha z}, \\
\phi_{27 \alpha}= & 140 c_{\alpha x}-117 c_{\alpha}{ }^{2} c_{\alpha x}+27 c_{\alpha}{ }^{4} c_{\alpha x}-26 c_{\alpha x}{ }^{3}, \\
\phi_{28 \alpha}= & 140 c_{\alpha y}-117 c_{\alpha}{ }^{2} c_{\alpha y}+27 c_{\alpha}{ }^{4} c_{\alpha y}-26 c_{\alpha y}{ }^{3}, \\
\phi_{29 \alpha}= & 140 c_{\alpha z}-117 c_{\alpha}{ }^{2} c_{\alpha z}+27 c_{\alpha}{ }^{4} c_{\alpha z}-26 c_{\alpha z}{ }^{3}, \\
\phi_{30 \alpha}= & -368+900 c_{\alpha}{ }^{2}-505 c_{\alpha}{ }^{4}+77 c_{\alpha}{ }^{6}, \\
\phi_{31 \alpha}= & -802 c_{\alpha}{ }^{2}+747 c_{\alpha}{ }^{4}-137 c_{\alpha}{ }^{6}+2406 c_{\alpha x}{ }^{2} \\
& -2241 c_{\alpha}{ }^{2} c_{\alpha x}{ }^{2}+411 c_{\alpha}{ }^{4} c_{\alpha x}{ }^{2}, \\
\phi_{32 \alpha}= & 802 c_{\alpha y}{ }^{2}-747 c_{\alpha}{ }^{2} c_{\alpha y}{ }^{2}+137 c_{\alpha}{ }^{4} c_{\alpha y}{ }^{2}-802 c_{\alpha z}{ }^{2} \\
& +747 c_{\alpha}{ }^{2} c_{\alpha z}{ }^{2}-137 c_{\alpha}{ }^{4} c_{\alpha z}{ }^{2}, \\
\phi^{2} & 1002 c_{\alpha}{ }^{2}+1147 c_{\alpha}{ }^{4}-438 c_{\alpha}{ }^{6}+53 c_{\alpha}{ }^{8},
\end{array}
$$

and the equilibrium moments are as follows:

$$
\begin{aligned}
& \tilde{f}_{1}^{\text {(eq) }}=\rho, \\
& \tilde{f}_{2}^{\text {(eq) }}=\rho u_{x}, \\
& \tilde{f}_{3}^{\text {(eq) }}=\rho u_{y}, \\
& \tilde{f}_{4}^{\text {(eq) }}=\rho u_{z}, \\
& \tilde{f}_{5}^{\text {(eq) }}=\rho\left(11 u^{2}+33 T-26\right), \\
& \tilde{f}_{6}^{\text {(eq) }}=-\rho\left(-2 u_{x}^{2}+u_{y}^{2}+u_{z}^{2}\right), \\
& \tilde{f}_{7}^{\text {(eq) }}=\rho\left(u_{y}^{2}-u_{z}^{2}\right), \\
& \tilde{f}_{8}^{\text {(eq) }}=\rho u_{x} u_{y}, \\
& \tilde{f}_{9}^{\text {(eq) }}=\rho u_{x} u_{z},
\end{aligned}
$$




$$
\begin{aligned}
& \tilde{f}_{10}^{(\mathrm{eq})}=\rho u_{y} u_{z}, \\
& \tilde{f}_{11}^{(\mathrm{eq})}=\rho u_{x}\left(13 u^{2}+65 T-37\right), \\
& \tilde{f}_{12}^{(\mathrm{eq})}=\rho u_{y}\left(13 u^{2}+65 T-37\right), \\
& \tilde{f}_{13}^{(\mathrm{eq})}=\rho u_{z}\left(13 u^{2}+65 T-37\right), \\
& \tilde{f}_{14}^{(\mathrm{eq})}=\rho u_{x}\left(u_{y}^{2}-u_{z}^{2}\right) \text {, } \\
& \tilde{f}_{15}^{(\mathrm{eq})}=-\rho u_{y}\left(u_{x}^{2}-u_{z}^{2}\right) \text {, } \\
& \tilde{f}_{16}^{(\mathrm{eq})}=\rho u_{z}\left(u_{x}^{2}-u_{y}^{2}\right) \text {, } \\
& \tilde{f}_{17}^{(\mathrm{eq})}=-\rho u_{x}\left(7 u_{x}^{2}+45 u_{y}^{2}+45 u_{z}^{2}+111 T-55\right), \\
& \tilde{f}_{18}^{(\mathrm{eq})}=-\rho u_{y}\left(45 u_{x}^{2}+7 u_{y}^{2}+45 u_{z}^{2}+111 T-55\right) \text {, } \\
& \tilde{f}_{19}^{(\mathrm{eq})}=-\rho u_{z}\left(45 u_{x}^{2}+45 u_{y}^{2}+7 u_{z}^{2}+111 T-55\right) \text {, } \\
& \tilde{f}_{20}^{(\mathrm{eq})}=\rho u_{x} u_{y} u_{z} \text {, } \\
& \tilde{f}_{21}^{(\mathrm{eq})}=\rho\left(1035 T^{2}+690 T u^{2}\right. \\
& \left.-975 T+69 u^{4}-325 u^{2}+304\right), \\
& \tilde{f}_{22}^{(\mathrm{eq})}=-\rho\left(-2 u_{x}^{2}+u_{y}^{2}+u_{z}^{2}\right)\left(19 u^{2}+133 T-69\right) \text {, } \\
& \tilde{f}_{23}^{(\mathrm{eq})}=\rho\left(u_{y}^{2}-u_{z}^{2}\right)\left(19 u^{2}+133 T-69\right) \text {, } \\
& \tilde{f}_{24}^{(\mathrm{eq})}=\rho u_{x} u_{y}\left(3 u^{2}+21 T-8\right), \\
& \tilde{f}_{25}^{(\mathrm{eq})}=\rho u_{x} u_{z}\left(3 u^{2}+21 T-8\right), \\
& \tilde{f}_{26}^{(\mathrm{eq})}=\rho u_{y} u_{z}\left(3 u^{2}+21 T-8\right), \\
& \tilde{f}_{27}^{(\mathrm{eq})}=0 \text {, } \\
& \tilde{f}_{28}^{(\mathrm{eq})}=0 \text {, } \\
& \tilde{f}_{29}^{(\mathrm{eq})}=0 \text {, } \\
& \tilde{f}_{30}^{(\mathrm{eq})}=0 \text {, } \\
& \tilde{f}_{31}^{(\mathrm{eq})}=0 \text {, } \\
& \tilde{f}_{32}^{(\mathrm{eq})}=0 \text {, } \\
& \tilde{f}_{33}^{(\mathrm{eq})}=0 \text {. }
\end{aligned}
$$

\section{APPENDIX B: LINEAR STABILITY THEORY OF THE LATTICE BOLTZMANN MODEL}

Here we briefly reproduce the linear stability theory, introduced by Lallelamand and Luo. For a more extensive treatment of the stability theory, the reader is referred to the original reference [32]. The starting point is assuming that $f_{\alpha}$ equals a uniform and steady background $f_{\alpha}^{(0)}$ with a superimposed nonuniform perturbation $f_{\alpha}^{(1)}$. The equilibrium background corresponds to a mass density $\rho_{0}=1$, a fluid velocity $\boldsymbol{u}_{0}$, and a temperature $T_{0}$. Inserting $f_{\alpha}=f_{\alpha}^{(0)}+f_{\alpha}^{(1)}$ into Eq. (2) and using Eq. (5) for the collision matrix gives the following equation for the behavior of $f_{\alpha}^{(1)}$ :

$$
\begin{aligned}
& f_{\alpha}^{(1)}\left(\boldsymbol{x}+\boldsymbol{c}_{\alpha}, t+1\right)-f_{\alpha}^{(1)}(\boldsymbol{x}, t) \\
& \quad=\phi_{\alpha \beta}^{-1} \tilde{\Lambda}_{\beta \gamma}\left(\left[\tilde{f}_{\gamma}^{(0)}+\tilde{f}_{\gamma}^{(1)}\right]^{(\mathrm{eq})}-\tilde{f}_{\gamma}^{(0)}-\tilde{f}_{\gamma}^{(1)}\right)(\boldsymbol{x}, t),
\end{aligned}
$$

where it is used that $f_{\alpha}^{(0)}$ is uniform and steady.
The next step is to linearize the equilibrium term,

$$
\left[\tilde{f}_{\alpha}^{(0)}+\tilde{f}_{\alpha}^{(1)}\right]^{(\mathrm{eq})}=\tilde{f}_{\alpha}^{(0)}+\tilde{E}_{\alpha \beta} \tilde{f}_{\beta}^{(1)}
$$

where it is used that $f_{\alpha}^{(0)}$ is at equilibrium. The matrix $\tilde{E}_{\alpha \beta}=$ $\left.\frac{\delta \tilde{f}_{\alpha}^{(\text {eq })}}{\delta \tilde{f}_{\beta}}\right|_{\tilde{f}=\tilde{f}^{(0)}}$ in Eq. (B2) is readily evaluated by rewriting the equilibrium moments $\tilde{f}_{\alpha}^{(\mathrm{eq})}$ from expressions in $\rho, \boldsymbol{u}$, and $T$ (see Appendix A) into expressions in terms of the five conserved moments $\tilde{f}_{1}, \tilde{f}_{2}, \tilde{f}_{3}, \tilde{f}_{4}$ and $\tilde{f}_{5}$. Combining Eqs. (B1) and (B2) gives:

$f_{\alpha}^{(1)}\left(\boldsymbol{x}+\boldsymbol{c}_{\alpha}, t+1\right)=\left[\delta_{\alpha \gamma}+\Lambda_{\alpha \beta}\left(E_{\beta \gamma}-\delta_{\beta \gamma}\right)\right] f_{\gamma}^{(1)}(\boldsymbol{x}, t)$,

where $\delta_{\alpha \beta}$ is the unit matrix. It is noted that in Eq. (B3) the matrices are evaluated in velocity space.

The final step in the linear stability theory is to decompose $f_{\alpha}^{(1)}$ into its Fourier components $\hat{f}_{\alpha}^{(1)}$,

$$
f_{\alpha}^{(1)}(\boldsymbol{x}, t)=\hat{f}_{\alpha}(\boldsymbol{k}, t) \exp (i \boldsymbol{k} \cdot \boldsymbol{x}) .
$$

Inserting Eq. (B4) into Eq. (B3) leads to the following evolution equation for the wave amplitude:

$$
\hat{f}_{\alpha}(\boldsymbol{k}, t+1)=L_{\alpha \beta}(\boldsymbol{k}) \hat{f}_{\beta}(\boldsymbol{k}, t),
$$

where $L_{\alpha \beta}$ is the linearized evolution operator:

$$
L_{\alpha \beta}=F_{\alpha \gamma}\left(\delta_{\gamma \beta}+\Lambda_{\gamma \delta}\left[E_{\delta \beta}-\delta_{\delta \beta}\right]\right),
$$

and $F_{\alpha \beta}$ contains $\exp \left(-i \boldsymbol{k} \cdot \boldsymbol{c}_{\alpha}\right)$ on its diagonal.

\section{APPENDIX C: THE EFFECT OF FILTERING ON THE HYDRODYNAMICS}

Here we outline the so-called Chapman-Enskog expansion to derive the equations of motion for mass, momentum, and energy and demonstrate that filtering the second- and third-order collision moments in the LB equation acts as diffusing the nonequilibrium momentum and heat fluxes. For an extensive treatment of the Chapman-Enskog expansion in the LB method, the reader is referred to Ref. [4]. Unlike the rest of this paper, we do not assume summation over repeated indices in this Appendix. Instead, we write summation symbols explicitly.

The Chapman-Enskog expansion is based on expanding the distribution function around the equilibrium as a function of the small parameter Knudsen number $\epsilon$ :

$$
f_{\alpha}=f_{\alpha}^{(\mathrm{eq})}+\epsilon f_{\alpha}^{(1)}+\epsilon^{2} f_{\alpha}^{(2)}
$$

applying a multiscale expansion for time and spatial scales:

$$
\partial_{t}=\epsilon \partial_{t_{0}}+\epsilon^{2} \partial_{t_{1}}, \quad \nabla=\epsilon \nabla,
$$

Taylor expanding the streaming operator:

$$
\begin{aligned}
& f_{\alpha}\left(\boldsymbol{x}+\boldsymbol{c}_{\alpha}, t+1\right)-f_{\alpha}(\boldsymbol{x}, t) \\
& \quad=\left[\partial_{t}+\boldsymbol{c}_{\alpha} \cdot \nabla+\frac{1}{2}\left(\partial_{t}+\boldsymbol{c}_{\alpha} \cdot \nabla\right)^{2}\right] f_{\alpha}(\boldsymbol{x}, t)
\end{aligned}
$$

and Taylor expanding the filtered collision operator:

$$
\begin{aligned}
\sum_{\boldsymbol{y}, \beta, \gamma} & {\left[\delta_{\alpha \beta} \delta(\boldsymbol{y})+v^{F} N_{\alpha \beta} \psi(\boldsymbol{y})\right] \Lambda_{\beta \gamma} } \\
\times & {\left[f_{\gamma}^{(\mathrm{eq})}(\boldsymbol{x}+\boldsymbol{y}, t)-f_{\gamma}(\boldsymbol{x}+\boldsymbol{y}, t)\right] } \\
= & \sum_{\beta} M_{\alpha \beta}\left[f_{\beta}^{(\mathrm{eq})}(\boldsymbol{x}, t)-f_{\beta}(\boldsymbol{x}, t)\right] .
\end{aligned}
$$


Here the effective collision operator is given by:

$$
M_{\alpha \gamma}=\sum_{\beta}\left(\delta_{\alpha \beta}+N_{\alpha \beta} v^{F} \nabla^{2}\right) \Lambda_{\beta \gamma},
$$

where we have used that Eq. (10) is the second-order discrete representation of the Laplacian,

$$
\sum_{\boldsymbol{y}} \psi(\boldsymbol{y}) f(\boldsymbol{x}+\boldsymbol{y})=\nabla^{2} f(\boldsymbol{x}) .
$$

In the moment space $\tilde{N}_{\alpha \beta}$ has unit diagonal elements for nonconserved second- and third-order moments and it is zero elsewhere. Therefore in the moment space $\tilde{M}_{\alpha \beta}$ has $\frac{1}{\tau}\left(1+v^{F} \nabla^{2}\right)$ on the diagonal elements for the nonconserved second- and third-order moments, it has one on the diagonal elements for fourth- and higher-order moments, and it is zero elsewhere.

Using Eqs. (C1)-(C5) in Eq. (9) and collecting terms of order $\epsilon$ gives us the first-order approximation to the filtered LB equation,

$$
\left(\partial_{t_{0}}+\boldsymbol{c}_{\alpha} \cdot \nabla\right) f_{\alpha}^{(\mathrm{eq})}=-\sum_{\beta} M_{\alpha \beta} f_{\beta}^{(1)} .
$$

The first-order equations for the density of mass, momentum, and energy are obtained by multiplying Eq. (C7) with $1, \boldsymbol{c}_{\alpha}$, or $c_{\alpha}^{2}$, respectively, and summing the result over $\alpha$, which is equivalent to computing the conserved moments of Eq. (C7). In this procedure, we use Maxwell-Boltzmann equilibrium moments and zero conserved moments of the effective collision operator, which is equivalent to zero conserved moments of $f_{\alpha}^{(1)}$ and $f_{\alpha}^{(2)}$.

The second-order approximation to the filtered LB equation reads:

$$
\begin{aligned}
& \partial_{t_{1}} f_{\alpha}^{(\mathrm{eq})}+\frac{1}{2}\left(\partial_{t_{0}}+\boldsymbol{c}_{\alpha} \cdot \nabla\right)^{2} f_{\alpha}^{(\mathrm{eq})}+\left(\partial_{t_{0}}+\boldsymbol{c}_{\alpha} \cdot \nabla\right) f_{\alpha}^{(1)} \\
& \quad=-\sum_{\beta} M_{\alpha \beta} f_{\beta}^{(2)} .
\end{aligned}
$$

On taking conserved moments of this equation, the $f_{\alpha}^{(1)}$ term generates the following nonzero, nonequilibrium moments:

$$
\begin{aligned}
& \sum_{\alpha} \boldsymbol{c}_{\alpha} \boldsymbol{c}_{\alpha} f_{\alpha}^{(1)}=-\tau\left(1-v^{F} \nabla^{2}\right) \sum_{\alpha} \boldsymbol{c}_{\alpha} \boldsymbol{c}_{\alpha}\left(\partial_{t_{0}}+\boldsymbol{c}_{\alpha} \cdot \nabla\right) f_{\alpha}^{(\mathrm{eq})}, \\
& \sum_{\alpha} \boldsymbol{c}_{\alpha} c_{\alpha}^{2} f_{\alpha}^{(1)}=-\tau\left(1-v^{F} \nabla^{2}\right) \sum_{\alpha} \boldsymbol{c}_{\alpha} c_{\alpha}^{2}\left(\partial_{t_{0}}+\boldsymbol{c}_{\alpha} \cdot \nabla\right) f_{\alpha}^{(\mathrm{eq})} .
\end{aligned}
$$

These expressions are derived from Eq. (C7) in combination with the following eigenvalue relations of the effective collision operator:

$$
\begin{aligned}
& \sum_{\alpha} \boldsymbol{c}_{\alpha} \boldsymbol{c}_{\alpha} M_{\alpha \beta}=\frac{1}{\tau}\left(1+v^{F} \nabla^{2}\right) \boldsymbol{c}_{\beta} \boldsymbol{c}_{\beta}, \\
& \sum_{\alpha} \boldsymbol{c}_{\alpha} c_{\alpha}^{2} M_{\alpha \beta}=\frac{1}{\tau}\left(1+v^{F} \nabla^{2}\right) \boldsymbol{c}_{\beta} c_{\beta}^{2},
\end{aligned}
$$

and the following expression for the inverse of the filter function:

$$
\left(1+v^{F} \nabla^{2}\right)^{-1}=\left(1-v^{F} \nabla^{2}\right),
$$

which is valid for small $v^{F}$. By combining Eqs. (C7), (C8), and (C9), we arrive at the following second-order equations that describe the changes due to nonequilibrium momentum and heat fluxes:

$$
\begin{aligned}
\partial_{t_{1}} \sum_{\alpha} \boldsymbol{c}_{\alpha} f_{\alpha}^{(\mathrm{eq})}= & \nabla \cdot\left\{\left[\tau\left(1-v^{F} \nabla^{2}\right)-\frac{1}{2}\right]\right. \\
& \left.\times \sum_{\alpha} \boldsymbol{c}_{\alpha} \boldsymbol{c}_{\alpha}\left(\partial_{t_{0}}+\boldsymbol{c}_{\alpha} \cdot \nabla\right) f_{\alpha}^{(\mathrm{eq})}\right\}, \\
\partial_{t_{1}} \sum_{\alpha} c_{\alpha}^{2} f_{\alpha}^{(\mathrm{eq})}= & \nabla \cdot\left\{\left[\tau\left(1-v^{F} \nabla^{2}\right)-\frac{1}{2}\right]\right. \\
& \left.\times \sum_{\alpha} \boldsymbol{c}_{\alpha} c_{\alpha}^{2}\left(\partial_{t_{0}}+\boldsymbol{c}_{\alpha} \cdot \nabla\right) f_{\alpha}^{(\mathrm{eq})}\right\} .
\end{aligned}
$$

It is therefore concluded that, up to second order, the filtering replaces $\tau$ by $\tau\left(1-v^{F} \nabla^{2}\right)$ in the overall equations of fluid motion,

$$
\begin{aligned}
\frac{\partial \rho}{\partial t}= & -\nabla \cdot(\rho \boldsymbol{u}), \\
\frac{\partial \rho \boldsymbol{u}}{\partial t}= & -\nabla \cdot(\rho \boldsymbol{u} \boldsymbol{u}+\rho \boldsymbol{T} \boldsymbol{\delta}+\boldsymbol{\Pi}), \\
& \frac{\partial \rho\left(\frac{1}{2} u^{2}+\frac{D}{2} T\right)}{\partial t} \\
= & -\nabla \cdot\left[\rho \boldsymbol{u}\left(\frac{1}{2} u^{2}+\frac{D+2}{2} T\right)\right. \\
& \left.-\kappa\left(1-v^{F} \nabla^{2}\right) \nabla T+\boldsymbol{u} \cdot \boldsymbol{\Pi}\right], \\
\boldsymbol{\Pi}= & -\mu\left(1-v^{F} \nabla^{2}\right)\left(\nabla \boldsymbol{u}+(\nabla \boldsymbol{u})^{T}-\frac{2}{D} \boldsymbol{\delta} \nabla \cdot \boldsymbol{u}\right),
\end{aligned}
$$

with a dynamic viscosity of $\mu=\rho T\left(\tau-\frac{1}{2}\right)$, a thermal conductivity of $\kappa=\rho T c_{p}\left(\tau-\frac{1}{2}\right)$, a specific heat capacity at constant volume of $c_{v}=\frac{D}{2}$, a specific heat capacity at constant pressure of $c_{p}=c_{v}+1=\frac{D+2}{2}$, a specific heat capacity ratio of $\gamma=\frac{c_{p}}{c_{v}}=\frac{D+2}{D}$, a sound speed of $c_{s}=\sqrt{\gamma T}=\sqrt{\frac{D+2}{D} T}$, a kinematic viscosity of $v=\frac{\mu}{\rho}=T\left(\tau-\frac{1}{2}\right)$, a thermal diffusivity of $\alpha=\frac{\kappa}{\rho c_{p}}=T\left(\tau-\frac{1}{2}\right)$, and a Prandtl number of $\operatorname{Pr}=\frac{v}{\alpha}=1$.

\section{APPENDIX D: REFERENCE SOLUTION TO THERMAL POISEUILLE FLOW}

A gas with relaxation time $\tau$ and average density $\rho_{0}$ is driven in the $x$ direction by an external pressure gradient $-\partial_{x} P$ between two walls that are located at $y=-H / 2$ and $y=H / 2$ and that are kept at a constant temperature $T_{w}$. Viscous dissipation results in a temperature distribution between the walls. The fully developed system is described by the thermal Navier-Stokes equations [Eq. (C13)] supplemented with $-\partial_{x} P$. Under the present conditions these equations 
reduce to:

$$
\begin{aligned}
& 0=-\partial_{x} P+\left(\tau-\frac{1}{2}\right) \partial_{y}\left(\rho T \partial_{y} u_{x}\right), \\
& 0=-\partial_{y}(\rho T), \\
& 0=\partial_{y}\left[\rho T\left(\frac{5}{2} \partial_{y} T+u_{x} \partial_{y} u_{x}\right)\right],
\end{aligned}
$$

under the constraints that:

$$
\begin{aligned}
u_{x}(-H / 2)=u_{x}(H / 2) & =0, \\
T(-H / 2)=T(H / 2) & =T_{w}, \\
H^{-1} \int_{-H / 2}^{H / 2} \rho(y) d y & =\rho_{0} .
\end{aligned}
$$

The solution to Eqs. (D1a)-(D1f) reads:

$$
\begin{aligned}
u_{x} & =u_{0}\left(1-\eta^{2}\right), \\
T & =T_{w}+\frac{u_{0}^{2}}{5}\left(-1+2 \eta^{2}-\eta^{4}\right), \\
\rho & =\frac{p}{T},
\end{aligned}
$$

where $\eta=2 y / H$ and $u_{0}=-H^{2} \partial_{x} P /[8 p(\tau-1 / 2)]$. The pressure $p$, which is constant over $y$, is obtained by imposing
Eq. (D1f), which is solved numerically using built-in routines of the MATHEMATICA software.

\section{APPENDIX E: REFERENCE SOLUTION FOR THE STATIONARY RAYLEIGH-BÉNARD SETUP}

A gas with relaxation time $\tau$ and average density $\rho_{0}$ resides between a hot wall at $x=0$ with temperature $T_{h}$ and a cold wall at $x=H$ with temperature $T_{c}$. Gravitational acceleration $g$ points towards the hot wall in the negative $x$ direction.

The fully developed system is described by the thermal Navier-Stokes equations [Eq. (C13)] supplemented with the gravitational force $-\rho g$. Under the present conditions, these equations reduce to:

$$
\begin{aligned}
& 0=-\partial_{x}(\rho T)-\rho g, \\
& 0=-\partial_{x}\left(\rho T \partial_{x} T\right),
\end{aligned}
$$

under the constraints that

$$
\begin{aligned}
T(0) & =T_{h}, \\
T(H) & =T_{c}, \\
H^{-1} \int_{0}^{H} \rho(y) d y & =\rho_{0} .
\end{aligned}
$$

The solution to this system has been obtained numerically using built-in routines of the MATHEMATICA software.
[1] G. R. McNamara and G. Zanetti, Phys. Rev. Lett. 61, 2332 (1988).

[2] F. J. Higuera and S. Succi, Europhys. Lett. 8, 517 (1989).

[3] C. Cercignani, The Boltzmann Equation (Springer, Berlin, 1988).

[4] D. A. Wolf-Gladrow, Lattice-Gas Cellular Automata and Lattice Boltzmann Models: An Introduction (Springer, Berlin, 2000).

[5] S. Succi, The Lattice Boltzmann Equation: For Fluid Dynamics and Beyond (Clarendon, Oxford, 2001).

[6] G. McNamara and B. Alder, Physica A 194, 218 (1993).

[7] P. Pavlo, G. Vahala, L. Vahala, and M. Soe, J. Comput. Phys. 139, 79 (1998).

[8] X. He, S. Chen, and G. D. Doolen, J. Comput. Phys. 146, 282 (1998).

[9] T. Ihle and D. Kroll, Comput. Phys. Commun. 129, 1 (2000).

[10] P. Lallemand and L.-S. Luo, Phys. Rev. E 68, 036706 (2003).

[11] Y. Shi, T. S. Zhao, and Z. L. Guo, Phys. Rev. E 70, 066310 (2004).

[12] S. Chen, K. Luo, and C. Zheng, J. Comput. Phys. 231, 8278 (2012).

[13] I. V. Karlin, D. Sichau, and S. S. Chikatamarla, Phys. Rev. E 88, 063310 (2013).

[14] G. R. McNamara, A. L. Garcia, and B. J. Alder, J. Stat. Phys. 81, 395 (1995).

[15] M. Watari and M. Tsutahara, Phys. Rev. E 70, 016703 (2004).

[16] G. Gonnella, A. Lamura, and V. Sofonea, Phys. Rev. E 76, 036703 (2007).

[17] M. Sbragaglia and K. Sugiyama, Phys. Rev. E 82, 046709 (2010).
[18] T. Biciuşcă, A. Horga, and V. Sofonea, C. R. Mecanique 343, 580 (2015).

[19] G. R. McNamara, A. L. Garcia, and B. J. Alder, J. Stat. Phys. 87, 1111 (1997).

[20] D. N. Siebert, L. A. Hegele, Jr., and P. C. Philippi, Phys. Rev. E 77, 026707 (2008).

[21] X. Nie, X. Shan, and H. Chen, Phys. Rev. E 77, 035701 (2008).

[22] L. Biferale, F. Mantovani, M. Sbragaglia, A. Scagliarini, F. Toschi, and R. Tripiccione, Phys. Fluids 22, 115112 (2010).

[23] J. W. Shim, Phys. Rev. E 88, 053310 (2013).

[24] P. Ripesi, L. Biferale, S. F. Schifano, and R. Tripiccione, Phys. Rev. E 89, 043022 (2014).

[25] N. I. Prasianakis and I. V. Karlin, Phys. Rev. E 76, 016702 (2007).

[26] H. Chen, J. Stat. Phys. 81, 347 (1995).

[27] C. Teixeira, H. Chen, and D. M. Freed, Comput. Phys. Commun. 129, 207 (2000).

[28] N. Frapolli, S. S. Chikatamarla, and I. V. Karlin, Phys. Rev. E 90, 043306 (2014).

[29] N. Frapolli, S. S. Chikatamarla, and I. Karlin, J. Stat. Phys. 161, 1434 (2015).

[30] M. Geier, A. Greiner, and J. G. Korvink, Phys. Rev. E 73, 066705 (2006).

[31] V. Borue and S. A. Orszag, Phys. Rev. E 51, R856 (1995).

[32] P. Lallemand and L.-S. Luo, Phys. Rev. E 61, 6546 (2000).

[33] X. Shan, X.-F. Yuan, and H. Chen, J. Fluid Mech. 550, 413 (2006).

[34] X. Nie, X. Shan, and H. Chen, Europhys. Lett. 81, 34005 (2008) 
[35] H. Chen, I. Goldhirsch, and S. A. Orszag, J. Sci. Comput. 34, 87 (2008).

[36] G. Silva and V. Semiao, J. Comput. Phys. 269, 259 (2014).

[37] D. d'Humières, Philos. Trans. R. Soc. London A 360, 437 (2002).

[38] M. Bouzidi, D. d'Humières, P. Lallemand, and L.-S. Luo, J. Comput. Phys. 172, 704 (2001).
[39] H. Chen, P. Gopalakrishnan, and R. Zhang, Int. J. Mod. Phys. C 25, 1450046 (2014).

[40] G. A. Sod, J. Comput. Phys. 27, 1 (1978).

[41] R. M. Clever and F. H. Busse, J. Fluid Mech. 65, 625 (1974).

[42] F. Massaioli, R. Benzi, and S. Succi, Europhys. Lett. 21, 305 (1993). 